\title{
The investigation of myelomatosis by gel isoelectric focusing followed by electrophoresis
}

G. DALE, A. L. LATNER ${ }^{1}$, AND T. J. MUCKLE

From the Departments of Clinical Biochemistry and Pathology, the University of Newcastle upon Tyne-

SYNOPSIS The relatively simple technique of isoelectric focusing in polyacrylamide gel fol- $\overrightarrow{-}$ lowed by electrophoresis has been applied to the examination of sera and urines from patients $\frac{\mathbb{O}}{O}$ both proved and thought to be suffering from myelomatosis. The results obtained from this method correlate well with those obtained by immunoelectrophoresis.

Polyclonal gammopathies can be distinguished from the monoclonal variety and $\gamma \mathrm{G}$ and $\vec{\emptyset}$ $\gamma \mathrm{A}$ myeloma proteins may be readily differentiated from each other. Ig light chain proteins. can be demonstrated in both serum and urine.

The technique of isoelectric focusing in polyacrylamide gels followed by electrophoresis (Dale and Latner, 1969) provides a relatively simple and yet precise method for the investigation of protein changes in body fluids. The method involves the separation by isoelectric focusing of serum proteins in polyacrylamide gel cylinders in a manner similar to that described earlier (Dale and Latner, 1968). After this, the cylinders are embedded in a block of polyacrylamide gel containing Tris/ $\mathrm{HCl}$ buffer and subjected to electrophoresis at right angles to their longitudinal axes. In this way, a two-dimensional pattern is produced which can be used for the characterization of the serum proteins.

Received for publication 1 May 1969.

${ }^{1}$ Requests for reprints should be addressed to A.L.L. (Department of Clinical Biochemistry).
In regard to the immunoglobulins, $G$ and $A$ 을 are readily separated by this means and the $D$ technique should, therefore, be suited to the examination of serum and urine from cases of $\bar{N}$ myelomatosis. The present work was undertaken in order to correlate the findings in this disease with those obtained by immunoelectrophoresis.

\section{Methods}

Specimens of serum and urine from cases of myelomatosis were examined together with $a \stackrel{D}{?}$ number of sera which, on paper electrophoresis, $\stackrel{\mathbb{Q}}{\varnothing}$ gave protein patterns of the type found in that disease. The samples were each divided into two portions. One of us examined one of these by응 immunoelectrophoresis (Scheidegger, 1955) fol 
lowed by characterization of abnormal proteins with appropriate antisera either raised personally (Muckle, 1967), donated by Dr David Rowe (Birmingham), or obtained commercially (BaxterHyland, Norfolk; Hoechst Pharmaceuticals, London). Two of us examined the other portion by the two-dimensional technique involving isoelectric focusing (Dale and Latner, 1969). In the latter procedure, a standard volume of $5 \mu 1$ of serum or $20-40 \mu 1$ of urine (depending on the protein content) was used in each case. The results obtained from each technique were assessed separately. At this stage, the immunoelectrophoresis assessor did not know the findings of the others and vice versa. The results were then compared.

Fifty-five blood samples from 43 patients were examined by both procedures.

\section{Results}

The patterns obtained by the isoelectric focusing method fell into six main types:

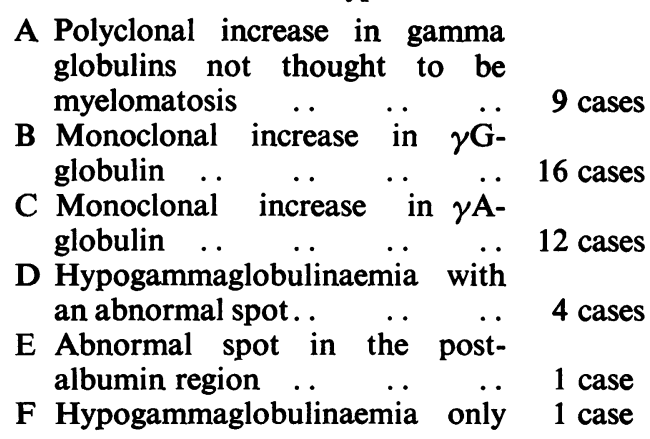

When more than one serum sample from the same patient was examined, excellent duplication was obtained.

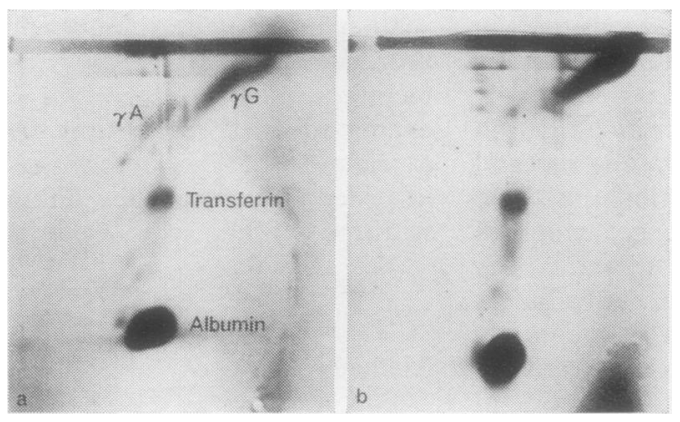

Fig. 1 Two-dimensional patterns. (a) Normal serum; the $\gamma A$ globulin area is defined by dots. (Note the elongated zone of $\gamma \mathrm{G}$ globulin); (b) serum showing polyclonal increase in $\gamma G$-globulin. The dark area in the bottom right hand corner is due to staining of the ampholine ampholytes.)
All the patients showing patterns of types $\bar{B}$ ? C, D, and E were eventually proved by clinicä and laboratory investigation to be suffering from? myelomatosis. In the majority of cases the diagnosis was confirmed at necropsy.

\section{TYPE A}

The results from the isoelectric focusing study agreed well with those from immunoelectro phoresis. Both techniques showed a polyclonal gammopathy in each case. A typical example of this type is shown in Fig. 1, in which the patter from the two-dimensional procedure is compared with that from a normal serum.

TYPE B

There was virtually complete agreement in the interpretation of the results of both techniques Immunoelectrophoresis suggested the presence of a trace of Ig light chain protein in one of the sera, but this was not demonstrable by the othef procedure. The two-dimensional patterns for the monoclonal increase in $\gamma \mathbf{G}$ globulin fell into tw categories (Fig. 2), one showing a localized in. crease in the $\gamma \mathrm{G}$-globulin area with decrease the remainder of the gamma globulins, and the other showing a well marked horizontal b. running out from the spot in the $\gamma$ G-globuli area towards the left of the gel.

TYPE C

The usual pattern obtained on isoelectric focusin of serum from cases of $\gamma \mathrm{A}$ myeloma is shown i Figure 3. In two cases, an unusual spot was demonstrated which migrated anodally to $\gamma \delta$ globulin during electrophoresis, but had virtualiy the same isoelectric point. A similar spot was

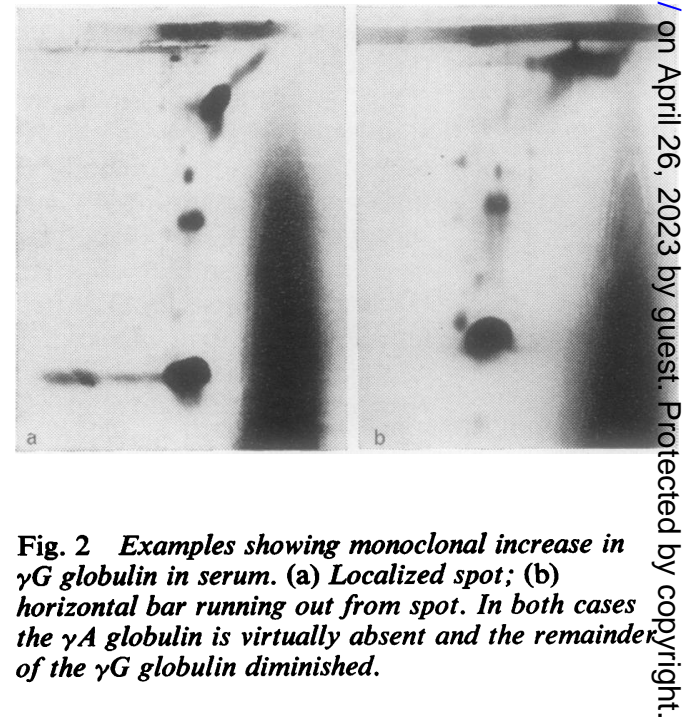



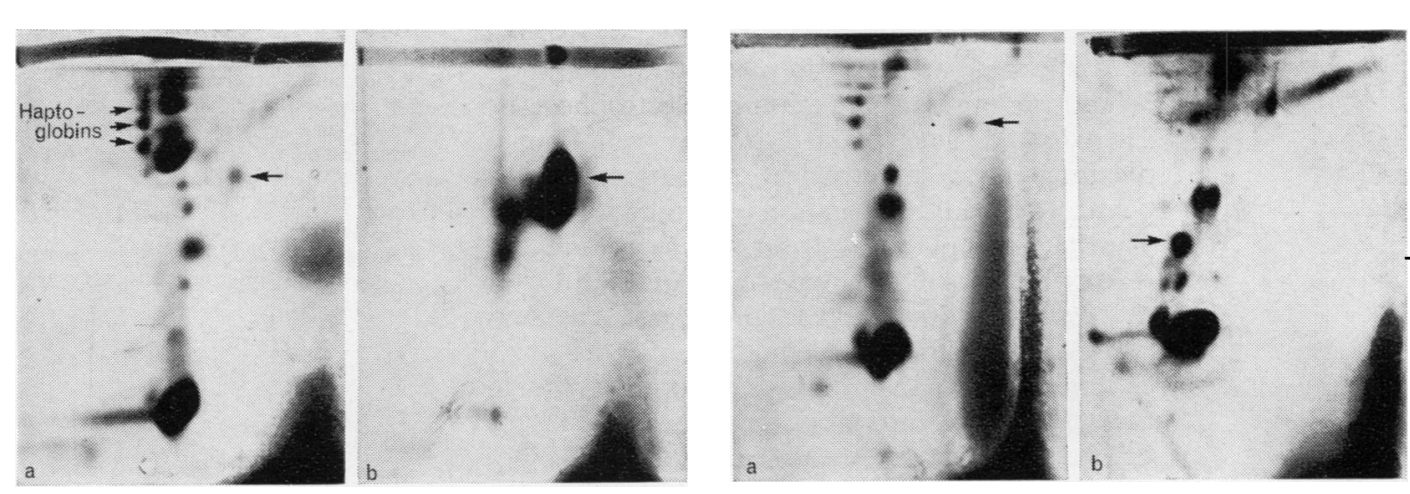

Fig. 3 Two-dimensional patterns showing (a) a monoclonal increase in $\gamma A$ globulin with markedly decreased $\gamma G$ globulin. Note that the $\gamma A$ globulin appears in the form of a series of spots. These are probably $\gamma$ A globulin polymers; (b) urine from the same patient. In each picture the unlabelled arrows denote Ig light chain. The serum pattern shows a well marked increase in haptoglobins.

found in each urine. Immunoelectrophoresis of the two sera showed the presence of Ig 'light chain proteins.

In only one case was there an apparent discrepancy between the result of immunoelectrophoresis and that of the two-dimensional gel procedure. This serum showed a gross reduction in the $\gamma \mathrm{G}$ globulin and a well marked spot which had a somewhat higher mobility during electrophoresis than expected for either $\gamma \mathrm{G}$ or $\gamma \mathrm{A}$ globulin. Because of its relatively acid isoelectric point, this had been judged to be $\gamma \mathrm{A}$ globulin, but immunological assessment showed it to be in fact $\gamma \mathrm{G}$ globulin.

In two cases with a monoclonal increase in $\gamma \mathrm{A}$ globulin, the two-dimensional gel technique revealed, in addition, a localized increase in the otherwise generally reduced $\gamma \mathrm{G}$ globulin.

TYPE D

Four of the cases examined by the two-dimensional gel procedure showed hypogammaglobulinaemia and the presence of an abnormal spot similar to that found in the two cases described in type $\mathrm{C}$ (Fig. 4). Immunoelectrophoresis of sera and urines from these cases demonstrated the presence of Ig light chain protein and confirmed the hypogammaglobulinaemia.
TYPE E

The single serum examined showed an unusual spot which, during electrophoresis, migrated in the post-albumin region (Fig. 4). Immunoelectrophoresis showed an Ig light chain protein with a similar mobility. Both techniques showed this to be present in the urine. In one of the cases of type $\mathrm{D}$ there was a suggestion of a similar spot in addition to the major abnormal one.

TYPE F

This picture was found in the serum obtained from a patient having macroglobulinaemia. The two-dimensional gel procedure showed only a diminution in both $\gamma \mathrm{G}$ and $\gamma \mathrm{A}$ globulins but immunoelectrophoresis demonstrated an increase in $\gamma \mathbf{M}$ globulin.

\section{Discussion}

From the results it would, therefore, appear that the two-dimensional gel technique (Dale and Latner, 1969) successfully discriminates between sera from patients with $\gamma \mathrm{G}$ and $\gamma \mathrm{A}$ myelomatosis. Sera from patients with $\gamma \mathrm{A}$ myelomatosis gave spots in a different position from those found in the sera from patients with $\gamma \mathrm{G}$ myelomatosis. 
These also differed in the tendency for the $\gamma \mathrm{A}$ to be associated with bands of protein migrating behind the normal $\gamma \mathrm{A}$ region on electrophoresis. These could be explained by the well known phenomenon of polymerization, but might also be due to complex formation with other proteins. The patterns obtained from the procedure are readily interpreted and have correlated well with the results of immunoelectrophoresis. The sensitivity of the former method of examination is certainly not less than that of the latter. Although they give similar interpretations, it would perhaps be best to regard these techniques as complementary. It is possible, however, that the two-dimensional gel technique more successfully indicates subgroups within the main $\gamma \mathrm{A}$ and $\gamma \mathrm{G}$ types of myelomatosis. Using this method, patients with $\gamma \mathrm{G}$ myelomatosis have sera corresponding to two types of pattern: either a single $\gamma \mathrm{G}$ spot with a decrease in the remainder of that band, or a spot associated with a bar running horizontally. We have not yet found an explanation for this latter phenomenon, but it may be due to complex formation between the $\gamma \mathrm{G}$ globulin and other proteins to form a larger molecule which does not migrate readily in the gel cylinder used for isoelectric focusing. Alternatively, it may be due to $\gamma \mathrm{G}$ polymers.

Judging from the results of immunoelectrophoresis, it seems likely that the unusual spots noted in the serum and urine of certain cases falling into types $C, D$, and $E$ were due to the presence of Ig light chain proteins. As demonstrated by the two-dimensional procedure, these light chain proteins fell into two groups, one with a mobility during electrophoresis akin to that of gamma globulin and the other migrating in the post-albumin zone.

Very large molecules, such as $\gamma \mathrm{M}$, do not appear to focus successfully under our conditions of isoelectric focusing. No doubt their migration is impeded by a molecular-sieve effect of the gel. It seems likely that the technique as at present used is not suitable for the investigation of increases in serum $\gamma \mathrm{M}$ globulin. The one case we investigated showed no abnormality of the gamma globulins other than a reduction in $\gamma \mathrm{G}$ and $\gamma \mathrm{A}$ globulins. It must be pointed out, however, that such a finding, taken together with the evidence from paper electrophoresis, might well prove useful in the diagnosis of macroglobulinaemia.

Polyclonal gammopathies can be easily distinguished from monoclonal increases in the gamma globulins. In addition, other protein changes can be demonstrated in myelomatosis; for example, several sera showed a quite marked increase in haptoglobins.
References

Dale, G., and Latner, A. L. (1968). Isoelectric focusing in poly acrylamide gels. Lancet $1,847-848$.

Dale, G., and Latner, A. L. (1969). Isoelectric focusing of seruff proteins in acrylamide gels followed by electrophores Clin. chim. Acta, 24, 61 .

Muckle, T. J. (1967). MD Thesis, University of Newcastle upom Tyne.

Scheidegger, J. J. (1955). Une micro-méthode de l'immunoélectrophorèse. Int. Arch. Allergy, 7, 103-110. 